\title{
Detection of Real Time QRS Complex Using Wavelet Transform
}

\author{
Fatima Yasmeen $^{1}$, M. A Mallick ${ }^{2}$, Y. U. Khan ${ }^{3}$ \\ ${ }^{1,2}$ Department of Electrical Engineering, Integral University, India \\ ${ }^{3}$ Department of Electrical Engineering, Aligarh Muslim University, India
}

\section{Article Info \\ Article history: \\ Received Dec 21, 2017 \\ Revised Apr 13, 2018 \\ Accepted May 8, 2018}

\section{Keyword:}

Electrocardiogram

QRS complex

RR interval

Wavelet transform

\section{Corresponding Author:}

Fatima Yasmeen,

Department of Electrical Engineering,

Integral University, Lucknow, India.

Email: has.fys@gmail.com

\begin{abstract}
This paper presents a novel method for QRS detection. To accomplish this task ECG signal was first filtered by using a third order Savitzky Golay filter. The filtered ECG signal was then preprocessed by a Wavelet based denoising in a real-time fashion to minimize the undefined noise level. R-peak was then detected from denoised signal after wavelet denoising. Windowing mechanism was also applied for finding any missing R-peaks. All the 48 records have been used to test the proposed method. During this testing, 99.97\% sensitivity and $99.99 \%$ positive predictivity is obtained for QRS complex detection.
\end{abstract}

Copyright (C) 2018 Institute of Advanced Engineering and Science. All rights reserved.

\section{INTRODUCTION}

Globally, various studies based on the incidence of cardiac failure are being conducted and according to an estimate one person dies every 33 seconds in India due to heart attack. The situation is quite alarming as the incidence of this disease is way high in India as compared to the developed nations. According to a study, an average Indian has to be on alert as the failure of the cardiac system is now more commonly observed in youngsters; and the figures show that more than two million heart attack cases are reported in a year [1].

The recording of bioelectrical activities of the cardiac system is known as the electrocardiogram (ECG) signals. It is an important source of information about the functional aspects of heart and cardiovascular system [2]-[6]. Timely detection of cardiovascular abnormalities and heart diseases not only reduces mortality rate but also increases life span with appropriate medication and recommended precautions. In this regard, various studies have been performed on the ECG signals [2]-[9]. The pattern of ECG and heart rate variability signal is indeed an important diagnostic parameter. However, as ECG monitoring is a lengthy and time consuming task, this increases the chances of error creeping in during data collection. Thus, in order to maximise the accuracy of analysis and further classification of the disease, a computerized approach is much more helpful in diagnostics. In conventional methods, monitoring and diagnosing electrocardiographic variation for appearance of specific signals depends on the reliability of human observation. Though this may be reliable when less number of samples are analysed, but for analysis of large number of patients in Cardiac Intensive Care Units (CICU) and Coronary Care units (CCU) of hospitals, this method would be inconsistent as conditions in such cases are critical and a thorough and minute observation is needed continuously.

Therefore, there is an urgent need to develop a more reliable computerised method to detect electrocardiographic changes. A reliable and specific quantitative signal feature classification problem is 
obtained through such automated techniques by transforming mostly qualitative diagnostic criteria. In some approach, the analysis of the ECG signals for detection of electrocardiographic changes is performed by using the autocorrelation function, principal component analysis, frequency domain features, time frequency analysis and empirical mode decomposition techniques [2]-[11]. In fact no matter how appropriate results are obtained by adopting such automatic techniques, there is a restriction with limited amount of information on electrocardiographic signal feature because of limitation of the underlying nonlinear signal dynamics [10]. In the recent past, considerable growth has been seen in application of nonlinear domain analysis and chaos theory in exploring the behaviour of a dynamical system from ECG signals in an experimental time series [10]-[16]. To remove the interference without deteriorating the original signal is not an easy task due to nonlinear signal dynamics. This can be overcome by using the modified variational mode decomposition and power line interference is removed satisfactorily [17]. The system performance for diagnosis of coronary heart disease was improved by using hybrid system of tiered multivariate analysis and artificial neural network and achieved an accuracy of 86.3\% [18]. Still processing of ECG signal needs a lot of improvement, most of all accurate detection of QRS complex. In this research paper, a novel computing method has been devised based on windowing mechanism to verify peak position combined with wavelet based de-noising for real time QRS detection.

\section{METHODOLOGY}

In this work R-peak has been detected from MIT BIH Arrhythmia data base. To carry out this task, raw ECG is first filtered by using third order Savitzky golay filter and then pre-processed by a wavelet based denoising in a real-time fashion to minimize the undefined noise level. R-peak is then detected from denoised signal after wavelet denoising. Windowing mechanism is also applied for finding the missing R-peaks if there is any. Descriptive data detailing of the above discussed signal processing stages are mentioned in following sections.

\subsection{Database Description}

The database of MIT-BIH Arrhythmia is incorporated in this work for the analysis of ECG signal. Beth Israel Hospital Arrhythmia Laboratory obtained a set of over 4000 long-term Holter recordings in the era of 1975 and 1979. To obtain these recordings, mainly the inpatients were analysed. There are 23 records in this database (ranges from 100 to 124 inclusive with some missing numbers data) chosen arbitrarily from this set, and 25 records (ranges from 200 to 234 inclusive, again with some numbers missing) selected from the same set to include a variety of rare but clinically important phenomena that would not be wellrepresented by a small random sample of Holter recordings. Each of the 48 records is slightly over 30 minutes long.

The first group is intended to serve as a representative sample of the variety of waveforms and artefact that an arrhythmia detector might encounter in routine clinical use. An arbitrary numbers were used for tapes selection, and then to select them for a segment of half-hour. If neither of the two ECG signals were of adequate quality for analysis by manual expertise are excluded in this approach.

The second group had chosen the records to include complicated supraventricular, junctional and ventricular arrhythmias and major abnormalities in conduction. Mostly, these records were selected because of QRS morphology variation, rhythm features or quality of signal might be expected to present difficulty to arrhythmia detectors in a significant manner; and considerable notoriety have recorded by database analyser. The study was conducted on 25 men aged 32 to 89 years, and 22 women aged 23 to 89 years. (Records 201 and 202 obtained from the same male subject).

\subsection{ECG Lead Configuration}

The electrodes are to be placed on chest for getting the upper signal which is a modified limb lead II (MLII). While a modified lead V1 (occasionally V2 or V5, and in one instance V4); in lower signals. In this case also, the electrodes are placed on the chest. BIH Arrhythmia Laboratory is using this configuration on regular basis. In the upper signals indications are generally prominent when QRS complex is normal. Mean cardiac electrical axis and the lead axis may be nearly orthogonal for the lower signal, however (i.e., normal beats are usually biphasic and may be nearly isoelectric). Thus ectopic beats are more illustrious in the lower signal, whereas normal beats are frequently difficult to perceive (see, for example, record 106).Reversed signals in record 114 making it a peculiar case for arrhythmia detectors as it often occurs in clinical practice. So arrhythmia analysers should be prepared to deal with such records. Modified lead V5 was used for the upper signal in records 102 and 104, as there was surgical dressings on the patients in these records so it was not advisable to use modified lead II [19]. 


\subsection{Wavelet Based Denoising Stage}

Along with the actual cardiac activity electrocardiogram signals are also comprised of several noise sources such as power-line interference, motion artifacts and baseline drifts. Initially, an appropriate signal pre-processing procedure for noise reduction is required for enhancement of QRS detection rate [20]. In this regard one of the most famous and common approach applied for this purpose is band pass filtering. But sometimes bandpass filtering could demolish intense features in electrocardiogram signal such as QRS complexes. For this reason researchers adopted wavelet based methods for biomedical signal processing. Temporal characteristics of a biomedical signal can be represented by unique components in frequency domain in wavelet transforms. Since wavelet transform based technique can also be used for signal denoising. In this preview of using application, a new method was developed by Donoho and johnstone which was named as wavelet shrinkage to denoise the signal with unknown noise characteristics while the intense features of the signal can be well maintained [21]. Since the QRS complex is an impulse-like waveform, however postulation might be possible in applying wavelet de-noising technique at the preprocessing stage for its viability and accuracy to the following stage of QRS detection.

We have also discussed in brief about procedure of de-noising scheme in our project. First of all ECG signal is filtered using a third order savitzky golay filter. These smoothing filters (also called digital smoothing polynomial filters or least-squares smoothing filters) are categorically used to "smooth out" a noisy signal where frequency span of signal (without noise) is large. The performance of Savitzky-Golay smoothing filters are far better than standard averaging FIR filters in such type of applications, which tend to filter out a remarkable portion of the signal's high frequency content along with the noise [22]

$$
\mathrm{B}=\text { sgolay }(\mathrm{k}, \mathrm{f}, \mathrm{w})
$$

Equation specifies a weighting vector $w$ with length $f$, which contains the real, positive-valued weights to be used during the least-squares minimization. Then, a Haar wavelet transform was performed with four-level decomposition in the measured ECG signal that has been filtered by Savitzky Golay filter. The Haar wavelet's mother wavelet function can be described as

$$
\begin{aligned}
\Psi(t) & =1 & & 0 \leq \mathrm{t} \leq \frac{1}{2}, \\
& =-1 & & \frac{1}{2} \leq \mathrm{t} \leq 1, \\
& =0 & & \text { otherwise }
\end{aligned}
$$

Scaling function of Haar Wavelet can be described as

$$
\begin{aligned}
\Phi(\mathrm{t}) & =1 & & 0 \leq \mathrm{t} \leq 1, \\
& =0 & & \text { otherwise. }
\end{aligned}
$$

\subsection{R-Peak Detection}

After denoising stage, the denoised signal is taken for R-peak detection and then detected R-peaks verified by considering three peaks window at a time. In window, the amplitude of second peak has been compared with other 2 peaks if its amplitude is less than $25 \%$, ignore the considered peak. If $R_{2} \geq 25 \%$ of $R_{1}$ or $R_{3}$, then $R_{2}$ is considered as peak where $R_{2}$ is the middle peak of the window, $R_{1}$ and $R_{3}$ are peaks on either side of the window.

\section{RESULTS AND DISCUSSION}

The proposed algorithm was tested on MIT BIH arrhythmia database using MATLAB codes. The sampling frequency of each recording in the database is $\mathrm{f}_{\mathrm{s}}=360 \mathrm{~Hz}$, with 11-bit resolution over $5 \mathrm{mV}$ range. The duration of each record is 30 min with 5.556s. Only the first channel of all 48 records has been considered for QRS detection. These records contain inverted QRS polarity, low amplitude QRS, ventricular ectopic beats with low SNR, premature ventricular beats, and premature atrial beats etc. The actual performance of the proposed algorithm has been evaluated by adopting two parameters: Sensitivity (Se), and positive predictivity $(+\mathrm{P})$, given below.

$$
\begin{aligned}
& \text { Se }(\%)=\frac{T P}{T P+F N} \times 100 \\
& +\mathrm{P}(\%)=\frac{T P}{T P+F P} \times 100
\end{aligned}
$$


Where, TP (True Positive) represents the number of heartbeats accurately recognized (i.e., QRS complexes accurately recognized), FN (False Negative) represents the number of heartbeats that were not recognized by the method (i.e., QRS complexes that were not recognized), and FP (False Positive) indicates the false heartbeats recognized (i.e., QRS complexes recognized by the method when no QRS complexes are present). The percentage of heartbeats that were correctly recognized by the algorithm are represented by sensitivity parameter (se) whereas, the percentage of real true heart-beats recognized by the algorithm are represented by positive predictivity $(+p)$.

The algorithm recognized the R-peak very accurately in some records that is even very close to the end of the record, e.g., record 100, 102, 112, 113, 115, 117, 122, 209, 212, 220, 230. The method acquired fairly good results also for very noisy records, e.g., record 108 (Figure 1). In addition, the method recognized precise results for record 203 (Figure 2) which is a very difficult record having considerable noise in both channels.

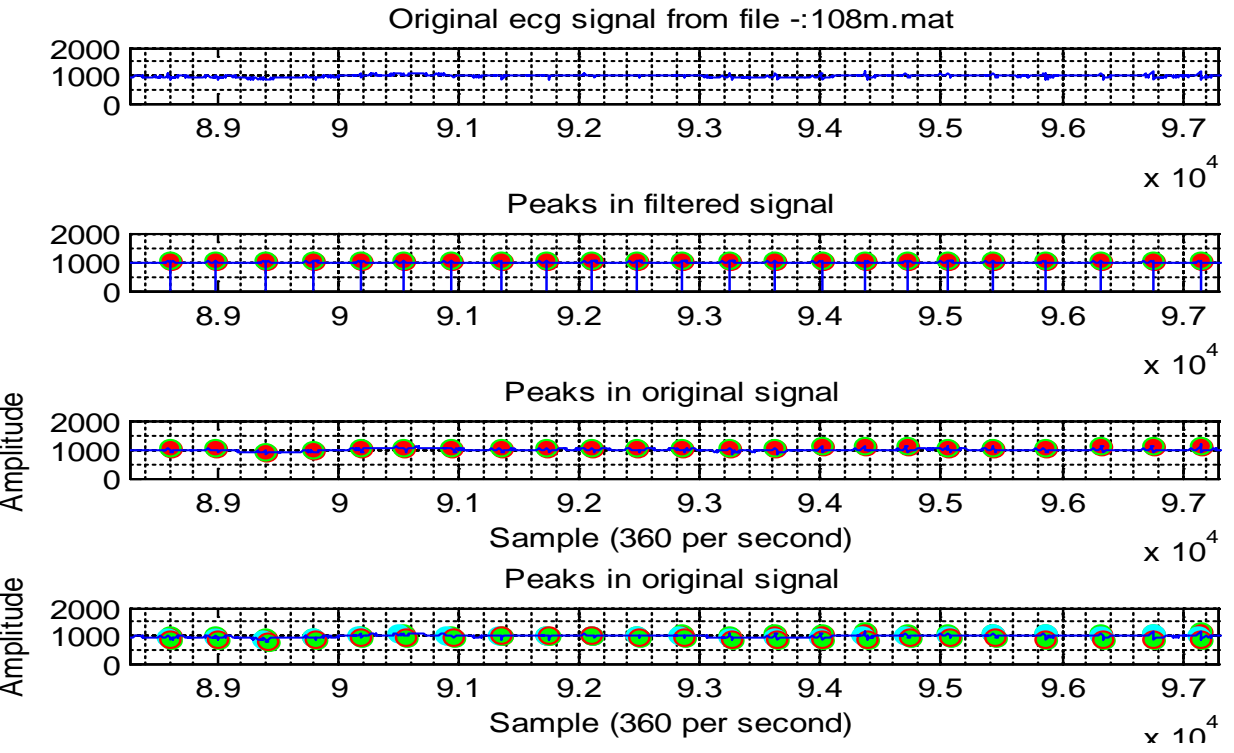

Figure 1. Graphs showing detected QRS complex in record 108 from MIT-BIH arrhythmia database

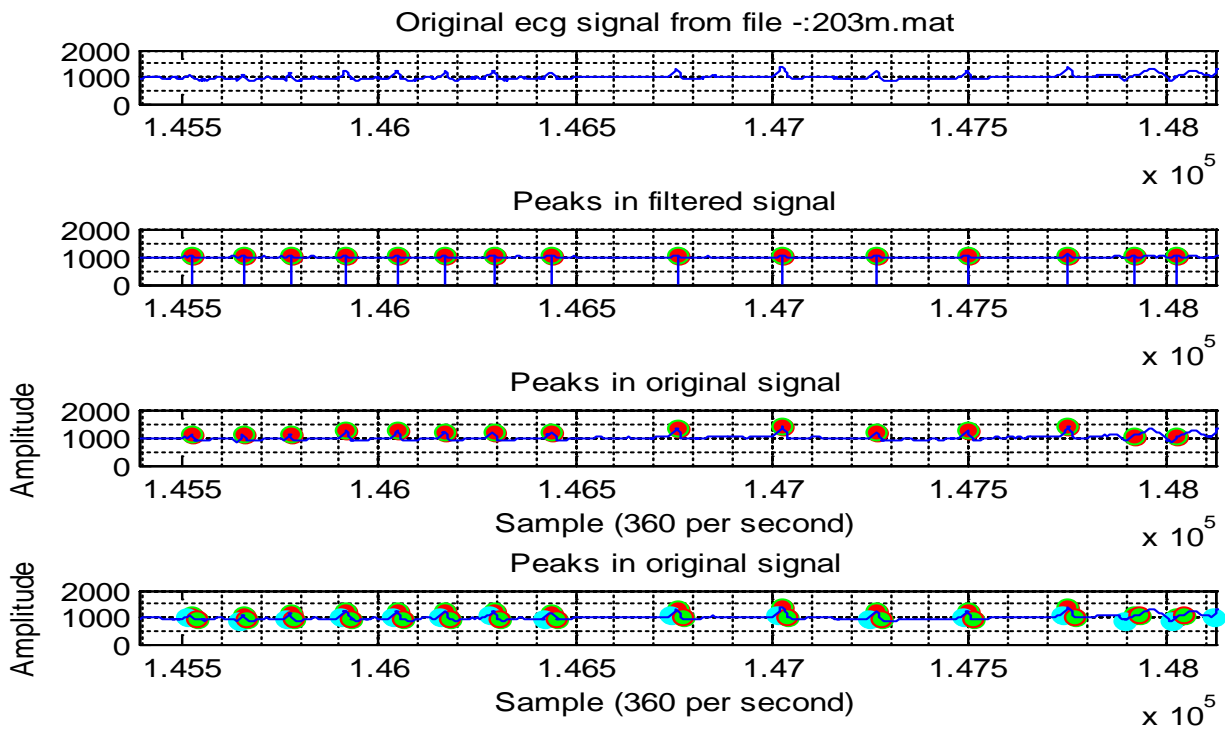

Figure 2. Graphs showing detected QRS complex in record 203 from MIT-BIH arrhythmia database 
The results of our method to detect QRS complexes are considerably accurate. Our method scored $\mathrm{Se}=99.97 \%$ and $+\mathrm{P}=99$. 99 over 109092 heartbeats, as shown in Table 1 . It is essential to acknowledge that our method acquired some less sensitivity rate for record 114 which presents supraventricular ectopics and ventricular ectopics as shown in Figure 3; this could explain the high number of FN beats, as can be seen in Table 1.

Table 1. Detected QRS Complex of MIT -BIH Arrhythmia Database

\begin{tabular}{ccccccc}
\hline Record & Beats & TP & FP & FN & Se & $+\mathrm{P}$ \\
\hline 100 & 2273 & 2273 & 0 & 0 & 100.00 & 100.00 \\
101 & 1865 & 1868 & 3 & 0 & 100.00 & 99.99 \\
102 & 2187 & 2187 & 0 & 0 & 100.00 & 100.00 \\
103 & 2084 & 2082 & 0 & 2 & 99.99 & 100.00 \\
104 & 2229 & 2217 & 0 & 12 & 99.98 & 100.00 \\
105 & 2572 & 2613 & 41 & 0 & 100.00 & 99.96 \\
106 & 2027 & 2023 & 0 & 4 & 99.99 & 100.00 \\
107 & 2137 & 2130 & 0 & 7 & 99.99 & 100.00 \\
108 & 1774 & 1830 & 56 & 0 & 100.00 & 99.94 \\
109 & 2532 & 2527 & 0 & 5 & 99.99 & 100.00 \\
111 & 2124 & 2122 & 0 & 2 & 99.99 & 100.00 \\
112 & 2539 & 2539 & 0 & 0 & 100.00 & 100.00 \\
113 & 1795 & 1795 & 0 & 0 & 100.00 & 100.00 \\
114 & 1879 & 1406 & 0 & 473 & 99.56 & 10.000 \\
115 & 1953 & 1953 & 0 & 0 & 100.00 & 100.00 \\
116 & 2412 & 2393 & 0 & 19 & 99.98 & 100.00 \\
117 & 1535 & 1535 & 0 & 0 & 100.00 & 100.00 \\
118 & 2288 & 2278 & 0 & 10 & 99.99 & 100.00 \\
119 & 1987 & 1988 & 1 & 0 & 100.00 & 99.99 \\
121 & 1863 & 1861 & 0 & 2 & 99.99 & 100.00 \\
122 & 2476 & 2476 & 0 & 0 & 100.00 & 100.00 \\
123 & 1518 & 1515 & 0 & 3 & 99.99 & 100.00 \\
124 & 1619 & 1608 & 0 & 11 & 99.98 & 100.00 \\
200 & 2601 & 2598 & 0 & 3 & 99.99 & 100.00 \\
201 & 2000 & 1886 & 0 & 114 & 99.89 & 100.00 \\
202 & 2136 & 2121 & 0 & 15 & 99.98 & 100.00 \\
203 & 2980 & 2920 & 0 & 60 & 99.94 & 100.00 \\
205 & 2656 & 2653 & 0 & 3 & 99.99 & 100.00 \\
207 & 2332 & 2193 & 0 & 139 & 99.87 & 100.00 \\
208 & 2955 & 2913 & 0 & 42 & 99.96 & 100.00 \\
209 & 3005 & 3005 & 0 & 0 & 100.00 & 100.00 \\
210 & 2650 & 2597 & 0 & 53 & 99.95 & 100.00 \\
212 & 2748 & 2748 & 0 & 0 & 100.00 & 100.00 \\
213 & 3251 & 3250 & 0 & 1 & 99.99 & 100.00 \\
214 & 2262 & 2259 & 0 & 3 & 99.99 & 100.00 \\
215 & 3363 & 3359 & 0 & 4 & 99.99 & 100.00 \\
217 & 2208 & 2206 & 0 & 2 & 99.99 & 100.00 \\
219 & 2287 & 2153 & 0 & 134 & 99.87 & 100.00 \\
220 & 2048 & 2048 & 0 & 0 & 100.00 & 100.00 \\
221 & 2427 & 2421 & 0 & 6 & 99.99 & 100.00 \\
222 & 2483 & 2471 & 0 & 12 & 99.98 & 100.00 \\
223 & 2605 & 2593 & 0 & 12 & 99.98 & 100.00 \\
228 & 2053 & 2050 & 0 & 3 & 99.99 & 100.00 \\
230 & 2256 & 2256 & 0 & 0 & 100.00 & 100.00 \\
231 & 1573 & 1571 & 0 & 2 & 99.99 & 100.00 \\
232 & 1780 & 1781 & 1 & 0 & 100.00 & 99.99 \\
233 & 3079 & 3071 & 0 & 8 & 99.99 & 100.00 \\
234 & 2753 & 2750 & 0 & 3 & 99.99 & 100.00 \\
Total & 110159 & 109092 & 102 & 1169 & 99.97 & 99.99 \\
\hline & & & & & &
\end{tabular}

Table 2 compares the performance of the proposed approach with other recent researches for the QRS detection over the MIT-BIH arrhythmia database. Chouakri et al quoted a sensitivity of 98.68\% and positive predictivity of $97.24 \%$ by applying a designed routine using haar and $\mathrm{db} 10$ based on histogram approach for the detection of QRS complex. Whereas Rodriguez et al applied Hilbert transform and adaptive threshold technique for QRS complex detection and reported a sensitivity of $96.28 \%$ and positive predictivity of $99.71 \%$. Our approach is simple to implement and performs much better as compared to the recent researches as shown in Table 2. 

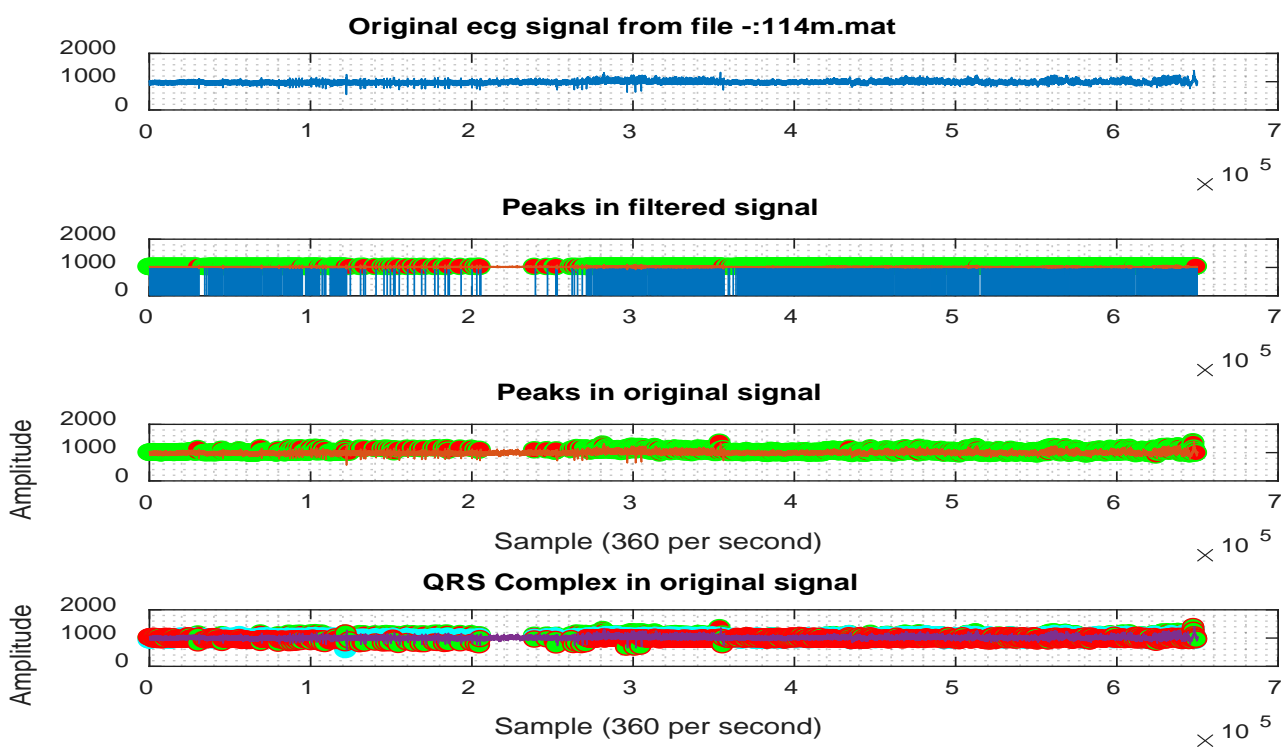

Figure 3. Graphs showing detected QRS Complex in record 114from MIT-BIH arrhythmia database

Table 2. Comparison of QRS Detection Performance on MIT-BIH Arrhythmia Database

\begin{tabular}{cccc}
\hline Work & Peaks & Se(\%) & $+\mathrm{P}(\%)$ \\
\hline Proposed & 109,092 & 99.97 & 99.99 \\
Rodriguez et al (2015) & 44,715 & 96.28 & 99.71 \\
Chouakri et al (2011) & 109,488 & 98.68 & 97.24 \\
\hline
\end{tabular}

\section{CONCLUSION}

This paper has presented a novel approach for QRS detection of electrocardiogram signals by applying the wavelet transform and windowing technique. Our approach improves the accuracy of QRS Complexes detection in records with different types of arrhythmias like Ventricular ectopics, Supraventricular ectopics, negative QRS Polarities, low signal to noise ratio etc. Our proposed approach achieves precise detection rates with an overall positive predictivity of $99.99 \%$ and a sensitivity of $99.97 \%$.

\section{ACKNOWLEDGEMENT}

The authors are thankful to Integral University for providing the infrastructure and manuscript communication number (IU/R\&D/2017-MCN000155) for this study. The authors are also thankful to Aligarh Muslim University.

\section{REFERENCES}

[1] A. Mehta, senior consultant cardiologist at Sir Ganga Ram Hospital, 2009.

[2] S. C. Saxena, et al., "Feature extraction from ECG signals using wavelet transforms for disease diagnostics," International Journal of Systems Science, vol/issue: 33(13), pp. 1073-1085, 2002.

[3] S. A. Chouakri, et al., "QRS complex detection based on multi wavelet packet decomposition," Applied Mathematics and Computation, vol. 217, pp. 9508-9525, 2011.

[4] S. Y. Foo, et al., "Neural network-based EKG pattern recognition," Engineering Applications of Artificial Intelligence, vol. 15, pp. 253-260, 2002.

[5] R. Rodriguez, et al., "Feature Extraction of Electrocardiogram Signalsby applying adaptive threshold and principal component analysis," Journal of Applied Research and Technology, vol. 13, pp. 261-269, 2015.

[6] N. Maglaveras, et al., "ECG pattern recognition and classification using non linear transformations and neural networks:a review,” International Journal of Medical Informatics, vol. 52, pp. 191-208, 1998.

[7] M. Kundu, et al., "Knowledge-based ECG interpretation: a critical review," Pattern Recognition, vol. 33, pp. 351373, 2000.

[8] K. Sternickel, "Automatic pattern recognition in ECG time series," Computer Methods and Programs in Biomedicine, vol. 68, pp. 109-115, 2002. 
[9] E. D. Übeyli, "Detection of electrocardiogram beats using a fuzzy similarity index," Expert Systems, vol/issue: 24(2), pp. 87-96, 2007.

[10] E. D. Übeyli, "Usage of eigenvector methods in implementation of automated diagnostic systems for ECG beats," Digital Signal Processing, vol/issue: 18(1), pp. 33-48, 2008.

[11] Fatima, et al., "Data Driven Approach for R-Peak Detection in Electrocardiogram (ECG) Signal,” International journal of advance Electrical and Electronics Engineering, pp. 2278-8948, 2015.

[12] M. I. Owis, et al., "Study of features based on nonlinear dynamical modeling in ECG arrhythmia detection and classification,” IEEE Transactions on Biomedical Engineering, vol/issue: 49(7), pp. 733-736, 2002.

[13] J. Fell, et al., "Nonlinear analysis of continuous ECG during sleep II. Dynamical measures," Biological Cybernetics, vol. 82, pp. 485-491, 2000.

[14] R. B. Govindan, et al., "On the evidence of deterministic chaos in ECG: surrogate and predictability analysis," Chaos, vol/issue: 8(2), pp. 495-502, 1998.

[15] R. Silipo, et al., "Dynamics extraction in multivariate biomedical time series,” Biological Cybernetics, vol. 79, pp. 15-27, 1998.

[16] A. Casaleggio and S. Braiotta, "Estimation of Lyapunov exponents of ECG time series-the influence of parameters,” Chaos, Solitons \& Fractals, vol/issue: 8(10), pp. 1591-1599, 1997.

[17] N. Mohan, "Modified Variational Mode Decomposition For Power Line Interference Removal in ECG Signals," IJECE, vol/issue: 6(1), 2016.

[18] Wiharto, et al., "Hybrid System of Tiered Multivariate Analysis and Artificial Neural Network for Coronary Heart Disease Diagnosis,” IJECE, vol/issue: 7(2), 2017.

[19] www.physionet.org

[20] S. W. Chen, et al., "A real-time QRS detection method based on moving-averaging incorporating with wavelet denoising," computer methods and programs in Biomedicine, vol. 82, pp. 187-195, 2006.

[21] D. L. Donoho and I. M. Johnstone, "Ideal spatial adaptation via wavelet shrinkage," Biometrika, pp. 425-455, 1994.

[22] S. J. Orfanidis, “Introduction to Signal Processing," Prentice-Hall, Englewood Cliffs, NJ, 1996. 